\title{
Deux examens du secondaire en France
}

Two examinations in the French system of education

Dos exámenes en el sistema educatica francesa

\section{Marc Fort}

\section{OpenEdition}

\section{Journals}

Édition électronique

URL : https://journals.openedition.org/ries/1381

DOI : $10.4000 /$ ries. 1381

ISSN : 2261-4265

Éditeur

France Education international

Édition imprimée

Date de publication : 1 décembre 2004

Pagination : 45-52

ISBN : 978-2-85420-564-0

ISSN : $1254-4590$

Référence électronique

Marc Fort, «Deux examens du secondaire en France », Revue internationale d'éducation de Sèvres [En ligne], 37 | décembre 2004, mis en ligne le 17 novembre 2011, consulté le 05 juillet 2021. URL : http:// journals.openedition.org/ries/1381; DOI : https://doi.org/10.4000/ries.1381 


\section{Deux examens du secondaire en France}

\section{Marc Fort}

Le système des principaux examens français est relativement simple dans son architecture. Le diplôme national du brevet (DNB) sanctionne la fin des études au collège, le baccalauréat la fin des études secondaires. Dans l'enseignement professionnel, le certificat d'aptitude professionnelle (CAP) et le brevet d'études professionnelles (BEP) sanctionnent différents niveaux de sorties, visant principalement l'insertion professionnelle. Les deux examens les plus importants numériquement et donc aussi par la place qu'ils occupent dans la société sont le diplôme national du brevet (à la session 2004, 791100 élèves) se sont présentés à cet examen et le baccalauréat où 602300 candidats se sont présentés en France métropolitaine à la session $2004^{1}$.

\section{LE BACCALAURÉAT : UN MÊME VOCABLE, DES EXAMENS DIFFÉRENTS}

Il existe trois types de baccalauréat, suivant le lycée fréquenté par le candidat. Le baccalauréat général pour les élèves des lycées d'enseignement général (organisé depuis 1993 en trois séries : économique et social (ES), littéraire (L) et scientifique (S), le baccalauréat technologique pour les élèves de lycées technologiques (organisé suivant huit séries : sciences et techniques industrielles (STI), sciences et technologie de laboratoire (STL), sciences médico-sociales (SMS), sciences et technologies tertiaires (STT), musique et danse (TMD); hôtellerie (HOT), sciences et techniques agricoles et environnementales (STAE) et sciences et techniques de l'agronomie et de l'environnement (STPA)) et enfin le baccalauréat professionnel (créé en 1985) pour les élèves de lycée professionnel (organisé en trois grands secteurs : production, services, services agricoles).

À la session 2004, les 602300 candidats qui se sont présentés se répartissaient de la manière suivante :

- baccalauréat général, 306959 élèves soit $51 \%$;

- baccalauréat technologique, 178872 élèves soit $30 \%$;

- baccalauréat professionnel, 116479 soit $19 \%$.

1. «Résultats provisoires du baccalauréat, France métropolitaine, session 2004 » Note d'information DEP, ministère de l'Éducation nationale, 04-19 juillet 2004. 
Ces données varient peu d'une année sur l'autre :

Nombre de présents au baccalauréat France métropolitaine et départements d'outre-mer

\begin{tabular}{|l|c|c|c|}
\hline & $\mathbf{2 0 0 1}$ & $\mathbf{2 0 0 2}$ & $\mathbf{2 0 0 3}$ \\
\hline Bac général & 326051 & 321548 & 320709 \\
\hline Bac technologique & 189535 & 184783 & 186171 \\
\hline Bac professionnel & 119424 & 112094 & 120640 \\
\hline Nombre total d'élèves & 635010 & 628425 & 627520 \\
\hline
\end{tabular}

En fin de classe de première, les candidats passent des épreuves dites «épreuves anticipées» dont systématiquement une épreuve de français (écrit et oral).

En fin de classe terminale, les candidats présentent des épreuves obligatoires écrites, orales, pratiques selon les séries, des épreuves facultatives, deux au maximum (où seuls les points supérieurs à 10 sont pris en compte par le jury).

À la fin de ce premier groupe d'épreuve, si le candidat a une moyenne inférieure à 8 sur 20, il est ajourné; si le candidat a obtenu une moyenne de $10 / 20$ et plus, il est définitivement admis; s'il a obtenu une moyenne comprise entre $8 / 20$ et $10 / 20$, il est autorisé à se présenter à des épreuves orales appelées «épreuves orales du second groupe» ou, plus communément «épreuves de rattrapage».

Le candidat se présente à deux épreuves orales dans deux matières qu'il choisit parmi celles qui ont été l'objet d'une épreuve écrite passée en première par anticipation ou en terminale. Seule la meilleure note obtenue par le candidat à l'épreuve du premier ou du second groupe est prise en compte par le jury. Le candidat est reçu s'il obtient, à l'issue de ces oraux, une moyenne de 10/20 ou plus à l'ensemble des épreuves. Un certificat de fin d'études secondaires est délivré au candidat ajourné à l'issue des épreuves du second groupe.

La responsabilité de l'organisation du baccalauréat incombe à quatre grands acteurs : l'administration centrale, l'inspection générale de l'éducation nationale, les services académiques, le chef du centre d'examen.

Selon les textes, l'administration centrale établit les textes réglementaires qui définissent le contenu de chacun des baccalauréats et les différentes modalités d'épreuves (durée, coefficient, nature, etc.), elle fixe le calendrier national des sessions, elle répartit la charge de l'élaboration des sujets entre les différentes académies et enfin fixe et répartit les emplois et les crédits nécessaires aux examens.

Les membres de l'inspection générale sont réglementairement responsables devant le ministre de la qualité des sujets du baccalauréat. Ils sont représentés au niveau académique par des inspecteurs territoriaux qui jouent un rôle important d'animation et de conseil tout au long de l'organisation de la session. 
Les services académiques sont eux, chargés de toute la logistique académique depuis la conception des sujets jusqu'à la conservation des copies pendant les douze mois qui suivent la session.

Le chef du centre d'examen est habituellement le proviseur de l'établissement qui accueille les candidats. Il est généralement assisté dans ses tâches d'un ou de plusieurs adjoints. Il est responsable du déroulement de l'examen dans l'établissement: préparation des salles, organisation de la surveillance, accueil des candidats, distribution des sujets, etc.

\section{L’archétype de l'examen français}

«Le baccalauréat est un monument national». Cette phrase a été prononcée par un ministre il y a quelques années à la suite des réactions provoquées par l'évocation de quelques réformes de cet examen. Elle résume parfaitement la situation : la puissance symbolique de cet examen dans la société française est telle qu'il est toléré «d'entretenir le monument» mais qu'il est hors de question de le modifier car il ne correspondrait plus à l'image mythique qu'il a dans la société française.

Cette difficulté à réformer le baccalauréat a deux conséquences.

Il est impossible de supprimer des épreuves alors que le nombre d'épreuves augmente régulièrement, rendant l'organisation de plus en plus lourde. Par exemple, pour la session 2004, le baccalauréat a commencé le 10 juin pour se terminer le 10 juillet : environ un mois! Pendant cette période, candidats, professeurs, encadrement des établissements se consacrent essentiellement à cette tâche alors que les travaux docimologiques montrent que le nombre d'épreuves pourrait être réduit et qu'ainsi, les élèves pourraient consacrer un peu plus de temps à apprendre.

Cette lourdeur grandissante de l'examen multiplie les risques d'erreurs. Un sursis à été donné par l'informatisation de certaines tâches, ce qui, paradoxalement a permis de rendre cet examen encore plus compliqué! On relira avec amusement le livre du Guy Bourgeois Le baccalauréat n'aura pas lieu ${ }^{2}$ où, dans un scénario catastrophe, l'auteur met bout à bout tous les incidents qui ne manquent pas de se produire chaque année.

La deuxième conséquence de cette impuissance à réformer fait que la modalité dominante d'évaluation, l'épreuve écrite ponctuelle finale, n'est pas toujours la mieux adaptée aux nouvelles compétences que l'on attend des élèves. Proposer d'autres modalités est quelquefois considéré comme un geste iconoclaste.

2. Le baccalauréat n'aura pas lieu, Guy Bourgeois, Payot, 1989. 
Examinons dans un premier temps ce qui caractérise cet examen : il est national, anonyme (donc équitable) et c'est le premier diplôme de l'enseignement supérieur.

\section{Paradoxes du baccalauréat}

Les épreuves sont nationales et en référence à des programmes nationaux. On peut donc admettre qu'il est national dans sa conception. Mais l'estil dans son déroulement et ses résultats? Les différences entre taux de réussite d'une académie à l'autre s'expliquent-elles uniquement par la qualité des enseignements dispensés?

La plus grande partie des épreuves étant des épreuves écrites ponctuelles finales rendues anonymes, le baccalauréat est un examen anonyme. Cet anonymat est, pour l'opinion, une garantie du caractère équitable de cet examen. Les tentatives d'introduction de nouvelles modalités d'examen risquant de briser cet anonymat sont systématiquement critiquées au nom de ce principe.

Cependant, dans d'autres pays, il paraîtrait inconcevable de porter un jugement sur les compétences d'un élève sans le connaître. Qui a tort, qui a raison?

Pour l'opinion, le caractère national et anonyme de l'examen garantit le fait qu'il est équitable. La disparité des taux de réussite d'une académie à l'autre devrait pourtant nous inciter à une certaine prudence vis-à-vis de cette affirmation. Mais, au sein d'une même académie, il peut y avoir aussi des disparités dues au grand nombre des jurys et à la faiblesse des dispositifs de régulation. L'informatisation du processus permet de disposer des séries de notes données par les différents jurys, une étude scientifique de leur répartition ne manquerait pas de surprendre.

\section{Le premier diplôme de l'enseignement supérieur}

Tout bachelier, qu'il soit titulaire d'un baccalauréat général, technologique ou professionnel a le droit de s'inscrire à l'université. De fait, l'admission dans un certain nombre de filières post-bac (classes préparatoires aux grandes écoles, DUT, BTS, certaines filières universitaires) se fait avant le baccalauréat, qui devient alors une simple formalité à accomplir pour intégrer ces formations. Il y a ainsi deux voies post-bac, celle sélective où l'étudiant est inscrit sur dossier avant l'obtention de l'examen, et celles où tout bachelier peut s'inscrire dans le cadre de numerus clausus plus ou moins maîtrisés.

\section{Qu'évalue-t-on au moment du baccalauréat?}

C'est une lapalissade que d'écrire qu'on n'évalue lors d'un examen que ce qu'on peut évaluer selon les modalités de cet examen. Il est évident qu'une épreuve ponctuelle finale ne permet pas de vérifier des compétences comme 
utiliser l'informatique pour résoudre un problème, mettre en place une démarche scientifique, etc.

Le choix des modalités n'est donc pas neutre, car ce qui est évalué au baccalauréat a un effet sur ce qui est enseigné les années précédentes. La responsabilité qu'ont les enseignants vis-à-vis de leurs élèves dans la réussite aux examens les incite à privilégier dans leur enseignement ce qui va accroître leurs chances de réussite. Les annales de sujets de baccalauréat seront encore pendant longtemps des succès de librairie! La question du choix de la modalité d'évaluation en fonction des objectifs poursuivis n'est jamais véritablement posée. Elle le mériterait certainement, car elle nous permettrait d'expliciter ce que nous attendons de l'enseignement au lycée et du baccalauréat.

Il y a ainsi une nette différence entre les objectifs de l'enseignement tels qu'ils sont affichés dans les programmes et ce qui est évalué au baccalauréat.

Il est intéressant de détailler le cheminement qui va des objectifs de l'enseignement tels qu'ils sont définis dans les programmes et ce qu'ont réellement acquis les élèves.

Partons des programmes, jugés insuffisamment explicites. L'institution leur adjoint des «documents d'accompagnement», qui sont parfois de véritables manuels. Dans une seconde étape, les auteurs de manuels s'emparent de ces programmes, les interprètent, les accommodent de sorte à garantir un tirage convenable : il faut plaire aux enseignants, aux élèves. Ensuite, il y a ce qui se passe dans la classe, l'enseignement que donne le professeur, ce que notent les élèves sur leurs cahiers ou classeurs (les différences peuvent être parfois surprenantes), ce qui est demandé au moment de l'examen. Et enfin, mystère parmi les mystères, ce que les élèves en ont retenu quelques années après leur sortie du lycée.

\section{LE DIPLÔME NATIONAL DU BREVET}

Le brevet d'études du premier cycle (BEPC), créé en 1948 pour sanctionner les études suivies dans les cours complémentaires et dans le premier cycle des collèges, a été remplacé par le brevet des collèges, puis en 1987 par le diplôme national du brevet. Celui-ci sanctionne la formation secondaire au terme des classes de troisième.

\section{Un examen sans conséquence}

Le diplôme national du brevet (DNB) est attribué aux élèves sur la base des notes obtenues à un examen comportant trois épreuves écrites ponctuelles finales en français, mathématiques, histoire - géographie - éducation civique, relatives à des sujets académiques (voire communs à plusieurs académies, non comparables et qui font l'objet «d'harmonisations» qui leur enlèvent une part 
de fiabilité) ainsi que sur la base des résultats acquis en cours de formation dans les classes de quatrième et de troisième.

Actuellement le brevet comporte trois séries (collège, technologique et professionnelle) qui sont adaptées à la formation donnée dans les classes de troisième ouvertes dans les collèges et les lycées professionnels.

Pour Michel Salines et Pierre Vrignaud ${ }^{3}$, dans leur rapport au Haut Conseil d'évaluation de l'école, «le brevet est pleinement l'affaire de l'inspecteur d'académie qui en assume personnellement et très activement la responsabilité, pour ce qui touche à l'organisation; la composition du jury; la gestion des résultats et des décisions de repêchage; la centralisation des notes, les synthèses, l'exploitation des résultats.»

Délivré par le jury, le brevet est un diplôme qui atteste de l'acquisition de compétences générales au terme du collège. Mais le brevet ne donne pas accès à une classe supérieure en fin de troisième. Les deux décisions, attribution du diplôme et orientation, sont dissociées. La décision d'orientation prend en compte les capacités spécifiques et les goûts des élèves pour la poursuite d'études. Elle est le résultat d'une négociation entre l'élève sa famille et l'équipe éducative. Les textes de 1987 et 1989 définissant l'examen du brevet ne prévoient aucune stratégie de mise en ouvre ou de suivi de leur application dans les académies, aucune remontée nationale des sujets des épreuves et des résultats chiffrés.

Le contrôle continu est ainsi mal régulé, dans un contexte où les enseignants des disciplines générales ont peu de pratique dans ce domaine. Aucune action de formation continue n'avait été prévue dans les textes au moment de la mise en place de cet examen.

La place du brevet dans la société française est de nature différente de celle du baccalauréat. Situé en fin de collège au terme de la classe de troisième, il est formellement indépendant de l'orientation de l'élève après cette classe et, en particulier, il ne conditionne pas le passage en classe de seconde.

\section{Des RÉsultats hétérogènes}

À la session 2003, le taux de réussite global pour le brevet a été de $78,0 \%$, en quasi-stabilité par rapport à la session 2002. Les résultats, tant au niveau académique que départemental, restent assez hétérogènes. Toutes séries confondues, il existe dix points d'écart entre les taux de réussite minimum et maximum de la session 2003.

Les académies qui réussissent le mieux dans la série collège ne sont pas forcément celles qui ont les meilleurs résultats dans les séries technologiques et professionnelles. C'est dans la série professionnelle que les écarts sont les plus élevés (33 points). Les écarts entre départements ont tendance à se creuser, l'écart maximal est de l'ordre de 20 points. Ils peuvent être également très

3. Voir bibliographie p. 52. 
prononcés entre les départements d'une même académie. Dans l'académie de Montpellier, 12 points séparaient le Gard et la Lozère.

Ces inégalités entre académies, départements, établissements ne peuvent s'expliquer par les seules différences de niveau des élèves. On n'est pas encore parvenu à une rigueur suffisante dans l'application des critères de notation définis de façon précise en fonction d'objectifs à atteindre et donc à une régulation suffisante du système de certification des acquis.

On éprouve le sentiment d'un examen marginalisé, privé de véritable considération et qui survit grâce au travail et à la considération des acteurs de terrain.

\section{A quoi sert donc le brevet?}

Comment l'indiquent Michel Salines et Pierre Vrignaud dans leur rapport, «l'acquisition des connaissances de base légitimement exigibles d'un citoyen à la fin de la scolarité obligatoire n'est que maladroitement assuré par cet examen pour plusieurs raisons. Les objectifs de fin de scolarité obligatoires ne sont pas définis de façon précise. De fait, tout se passe comme si on s'en remettait aux professeurs pour le faire. Dans ces conditions, il ne faut pas s'étonner que chacun agisse en fonction de ses propres exigences et parfois de façon strictement disciplinaire. Certains objectifs comportementaux, comme par exemple l'aptitude à l'expression, à la communication ne sont pas véritablement évalués, malgré leur rôle, aussi bien dans la poursuite d'études que dans la vie sociale et professionnelle.»

Ce constat, toujours d'actualité, appelle deux remarques. De même que pour le baccalauréat, nous ne disposons pas de moyens fiables pour déterminer les acquis des élèves en fin de scolarité obligatoire. Le brevet des collèges actuel n'en est certainement pas un. Peut-être faut-il miser sur des enquêtes internationales, comme PISA, par exemple? Ensuite, quels sont les savoirs, savoir faire, savoir être légitimement exigibles d'un citoyen à la fin de la scolarité obligatoire? Ce n'est peut-être pas au système éducatif de les définir, ces définitions relevant plus de la société ou du politique. Une fois définis, il appartient au système éducatif de s'organiser pour permettre l'atteinte de ces objectifs et leur évaluation. Il n'est pas certain que le modèle du diplôme national du brevet soit le plus pertinent pour cette évaluation. Dans tous les cas, l'évaluation en fin de scolarité obligatoire ne peut pas se réduire à la vérification de l'acquisition d'un «socle» de connaissances.

Le brevet peut-il être un outil de mesure du niveau de l'élève dans les différentes disciplines du collège? Alors qu'il «mobilise toutes les énergies, il fonctionne cependant $\mathrm{mal}^{4}$ : de fortes différences entre les notes de contrôle continu et les notes» aux épreuves ponctuelles finales mettant en doute la fiabi-

4. Ibid. 
lité des résultats, la faiblesse de la régulation entre disciplines au sein d'un même établissement, entre établissements, aussi bien au niveau départemental qu'au niveau académique, contribuent certainement à ces dysfonctionnements.

Les résultats des élèves au brevet ont peu d'intérêt en eux-mêmes, mais rapportés à d'autres indicateurs comme les catégories socio-professionnelles, les résultats à l'évaluation sixième en français et en mathématiques, ils peuvent contribuer au pilotage à tous les niveaux. Cela commence à être pratiqué, mais pourrait être développé et affiné. En tous les cas, ces pratiques sont loin encore d'être habituelles.

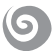

Le brevet des collèges et le baccalauréat sont deux examens parfaitement rôdés, inscrits dans une longue tradition, soixante ans pour le brevet, presque deux siècles pour le baccalauréat. L'analyse de leur fonctionnement montre cependant, que cette tradition gêne une évolution qui permettrait une meilleure prise en compte des objectifs de l'éducation. À ceci se rajoute un manque cruel de formation des enseignants dans le domaine de l'évaluation des élèves.

\section{BIBLIOGRAPHIE}

"Le système éducation français et son administration", 9e édition, Administration et éducation, septembre 2003.

«Le système éducatif en France», Les notices de la Documentation française, 2003.

BOURGEOIS Guy (1989): Le baccalauréat n'aura pas lieu, Payot.

«Résultats provisoires du baccalauréat, France métropolitaine, session 2004 », Note d'information, DEP, ministère de l'Éducation nationale, 0419 juillet 2004 .

«Résultats définitifs du diplôme national du brevet, session 2003 », Note d'information 04-10 avril 2004 [www. education. gouv. fr/stateval]

SALINES Michel, VRIGNAUD Pierre, "Apprécier et certifier les acquis des élèves en fin de collège : brevet et évaluations-bilans", Rapport établi à la demande du Haut Conseil de l'évaluation de l'école, juin 2001 [www.education.gouv.fr/hcee]

"L'évaluation des acquis des élèves à la fin des cycles des apprentissages ", Rapport de l'inspection générale de l'Éducation nationale, 1991. 